\section{PLA-3: A FORTRAN program for prediction logic analysis for three-dimensional contingency tables}

\author{
EDWARD E. BRENT, JR. \\ University of Missouri, Columbia, Missouri 65201
}

Description. This program is designed to provide measures of prediction logic for contingency table analysis as developed by Hildebrand, Laing, and Rosenthal (1977). The program computes a family of "del" $(\nabla)$ prediction logic measures that are proportionate reduction in error (PRE) measures of the extent to which specific predictions are upheld by the data. The general formula for the bivariate del measuring the prediction success of some specific prediction, $\rho$, in an $\mathrm{R}$ by $\mathrm{C}$ table is as follows:

$$
\nabla_{\rho}=1-\frac{\sum_{\mathrm{i}} \sum_{j} W_{\mathrm{ij}} \mathrm{P}_{\mathrm{ij}}}{\sum_{\mathrm{i}} \sum_{\mathrm{j}} \mathrm{W}_{\mathrm{ij}} \mathrm{P}_{\mathrm{i} .} \mathrm{P}_{\mathrm{j}}}
$$

where $P_{i j}, P_{i}$, and $P_{j}$ are the probabilities for Cell $i j$, Row $i$, and Column $j$, respectively; and the $W_{i j} S$ are the weights assigned to each cell corresponding to the specific prediction, $\rho$. These measures are not to be confused with traditional tests of statistical significance based on chi-square measures that test only whether there is any association between two variables without consideration of the form that relationship might take. The nature of prediction logic measures and their limitations is discussed by Goodman and Kruskal (1974a, 1974b) and Hildebrand, Laing, and Rosenthal (1974a, 1974b).

Either two- or three-dimensional contingency tables may be analyzed by this program. For each bivariate combination of variables, the program computes the bivariate del, the conditional bivariate del, measures of scope and precision for each, measures of the statistical significance of each, and the decomposition of each. The program also computes the trivariate del, its decomposition, and measures of the statistical significance for that coefficient. The program computes these coefficients for one or more specific predictions and weighting schemes provided by the user.

Options. The following options are available: (1) bivariate or trivariate analysis, (2) contingency table or raw data input, (3) multiple predictions and weighting schemes provided by the user.

Hardware and Software. This program is a standalone FORTRAN program requiring no other software than a FORTRAN IV compiler. The compil $\mathrm{r}$ uses the FORTRAN IV language as described in the publication, FORTRANIV Language (IBM Form GC28-6515). The program was developed on an IBM $370 / 168$ computer and the current version requires a memory of $300 \mathrm{~K}$.

Portability. The program should be portable to other IBM machines having sufficient storage. A CDC version is currently being developed.

Availability. Copies of the FORTRAN IV listing and write-up can be obtained at no charge by writing to Edward E. Brent, Department of Sociology, University of Missouri, Columbia, Missouri 65201.

\section{REFERENCES}

Goodman, L. A., \& Kruskal, W. Empirical evaluation of a formal theory. Journal of Mathematical Sociology, 1974, 3, 187-196. (a)

Goodman, L. A., \& KRUSKal, W. More about empirical evaluation of formal theory. Journal of Mathematical Sociology, 1974, 3, 211-213. (b)

Hildebrand, D. K., Laing, J. D., \& Rosenthal, H. Prediction logic: A method for empirical evaluation of formal theory. Journal of Mathematical Sociology, 1974, 3, 163-185. (a)

Hildebrand, D. K., Laing, J. D., \& Rosenthal, H. Prediction logic and quasi-independence in empirical evaluation of formal theory. Journal of Mathematical Sociology, 1974, 3, 197.209. (b)

Hildebrand, D. K., Laing, J. D., \& Rosenthal, H. Prediction analysis of cross classifications. New York: Wiley, 1977.

(Received November 18, 1977; revision accepted December $14,1977$. 